\title{
A pandemia de COVID-19 e suas repercussões na epidemia da obesidade de crianças e adolescentes
}

\author{
The COVID-19 pandemic and its impact on the obesity epidemic of children and teenagers \\ La pandemia del COVID-19 y sus repercusiones en la epidemia de la obesidad de niños y \\ adolescentes
}

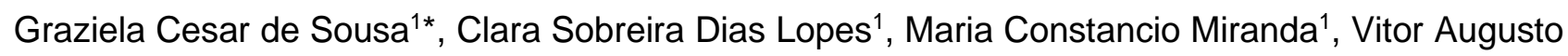
Alves da Silva1, Patrícia Regina Guimarães ${ }^{1}$.

\section{RESUMO}

Objetivo: Abordar os fatores implicados na gênese da obesidade, suas repercussões e estratégias para seu enfrentamento, além de elencar os possíveis impactos do distanciamento social decorrente da pandemia de COVID-19 na epidemia de obesidade infantil e juvenil. Revisão bibliográfica: O Brasil passou por uma rápida transição nutricional que culminou na diminuição percentual da desnutrição e despontamento da obesidade como importante problema de saúde pública. O caráter de sua distribuição e sua progressiva prevalência permitem caracterizar a obesidade como uma epidemia global. Entre as estratégias para enfrentá-la na população mais jovem estão o estímulo às atividades físicas, alimentação balanceada e limitação do tempo de exposição a aparelhos eletrônicos. O distanciamento social imposto pela epidemia de COVID-19 acarreta dificuldades para realização das estratégias de enfrentamento e estimula as práticas que podem desencadear ou agravar o excesso de peso, sendo potencialmente um complicador da epidemia de obesidade cujos reais impactos serão percebidos posteriormente. Considerações finais: As modificações nos hábitos de vida no que tange à restrição de atividades, alterações da dinâmica alimentar e aumento do tempo de utilização de eletrônicos, contribuem para um balanço energético positivo e, consequentemente, para maior possibilidade de surgimento ou agravamento de sobrepeso e obesidade em crianças e adolescentes.

Palavras-chave: Obesidade, Obesidade pediátrica, COVID-19, Comportamento sedentário.

\begin{abstract}
Objective: This article addresses the factors implicated in the genesis of obesity, its repercussions and presents strategies for coping with it, in addition to listing the possible impacts of social distancing indicated for the COVID-19 pandemic on the obesity epidemic among children and teenagers. Literature review: Brazil went through a rapid nutritional transition that culminated in a percentage decrease in malnutrition and obesity emergence as a serious public health problem. The characteristics of its distribution and its progressive prevalence allows us to characterize obesity as a global epidemic. Among the strategies to face the desease in the younger population, we can mention the encouragement of physical activities, a balanced diet, and limiting the time of exposure to electronic devices. Social distancing leads to difficulties in carrying out coping strategies and encourages practices that can trigger or aggravate excess weight, being potentially a complicator fot the obesity epidemic which its real impacts will be known later. Final considerations: Changes in life habits including activity restriction and changes in dietary dynamics associated with increased daily use of electronics, contribute to a positive energy balance and, consequently, to a greater possibility of the emergence or aggravation of overweight and obesity in children and teenagers.
\end{abstract}

Keywords: Obesity, Pediatric obesity, Coronavirus infections, Sedentary behavior.

${ }^{1}$ Pontifícia Universidade Católica de Minas Gerais (PUC-MG), Betim - MG.

*E-mail: graziela.cesar@sga.pucminas.br

SUBMETIDO EM: 7/2020

ACEITO EM: 8/2020

PUBLICADO EM: 12/2020 


\section{RESUMEN}

Objetivo: Abordar los factores implicados en la génesis de la obesidad, sus repercusiones y estrategias para enfrentarla, además de relacionar los posibles impactos del distanciamiento social ocasionados por la pandemia de COVID-19 en la epidemia de obesidad entre niños y adolescentes. Revisión bibliográfica: Brasil ha pasado por transición nutricional que ha culminado en la disminución porcentual de la desnutrición y surgimiento de la obesidad como un importante problema de la salud publica. Entre las estrategias para enfrentarla, en la población más joven, se encuentran el incremento de actividades físicas, una dieta equilibrada, y limitar el tiempo de exposición a dispositivos electrónicos. El distanciamiento social exigido por la epidemia del COVID-19 genera dificultades para la implementación de estrategias de afrontamiento y fomenta prácticas que pueden desencadenar o agravar la obesidad, siendo potencialmente un empeoramiento de la epidemia de obesidad cuyos impactos reales se percibirán más adelante. Consideraciones finales: Los cambios en los hábitos de la vida que implican la restricción de actividades y alteraciones en la dinámica alimenticia y aumento del tiempo de uso de dispositivos electrónicos, contribuyen para un equilibrio energético positivo y, en consecuencia, para una mayor probabilidad de surgimiento 0 incremento de sobrepeso y obesidad en niños y adolescentes.

Palabras clave: Obesidad, Obesidad pediátrica, Infecciones por coronavirus, Conducta sedentaria.

\section{INTRODUÇÃO}

A alimentação é um processo complexo cujo significado é permeado por fatores que extrapolam a simples necessidade de nutrir. A essência da alimentação e os significados que assume em cada contexto histórico são amplamente influenciados por valores culturais e sociais. Há um consenso no que tange ao significado simbólico e cultural da alimentação que assume elevada importância em diversos aspectos da dinâmica social e compreende um patamar próprio do que é sagrado. Esse entendimento da alimentação como algo imprescindível às funções humanas é tão profundo que ela foi incluída como direito social no artigo $6^{\circ}$ da Constituição Federal através da Emenda Constitucional no 64 de 2010, um reflexo da preocupação da sociedade brasileira com a garantia da subsistência nacional (BRASIL, 2013).

Uma análise histórica dos padrões alimentares no Brasil permite divisar uma transição da elevada prevalência da desnutrição para um cenário em que predominam o sobrepeso e a obesidade. Essa tendência de elevação percentual do excesso de peso é uma característica mundial, englobando todas as idades, ambos os sexos e constituindo importante problema de saúde pública em diversos países. Por seu caráter ubíquo, a obesidade é referida como uma epidemia global cujo enfrentamento é urgente e complexo, exigindo um comprometimento intersetorial e uma abordagem integral (BRASIL, 2006).

Esse enfrentamento se mostra tão premente que a Organização das Nações Unidas destaca, em seus Objetivos de Desenvolvimento Sustentável, a imprescindibilidade de alcançar a segurança alimentar e melhoria da nutrição, além de reduzir a mortalidade por doenças não transmissíveis e promover bem-estar, fatores intrinsecamente relacionados ao enfrentamento da obesidade (ONU, 2015).

A obesidade é caracterizada como o acúmulo exacerbado de gordura corporal resultante de um balanço energético positivo que acarreta danos à saúde dos indivíduos e provoca prejuízos em diversos âmbitos do funcionamento físico e psíquico. Apresenta-se, concomitantemente, como uma morbidade e como um fator de risco para outras doenças não transmissíveis, e sua prevalência assume trajetória ascendente na população brasileira. Nesse contexto, o acometimento cada vez mais precoce dos indivíduos desponta como um fenômeno de grande relevância. A obesidade em crianças e adolescentes traz diversas consequências ao desenvolvimento saudável e ao bem-estar, inclusive a longo prazo (BRASIL, 2006).

No Brasil, de acordo com o VIGITEL realizado em 2018, a frequência de sobrepeso entre os adultos foi de $55,7 \%$ e a obesidade apresentou uma frequência de 19,8\% (BRASIL, 2019). Em relação à obesidade infantil, na faixa etária de 5 a 9 anos, foi encontrada prevalência de $33,5 \%$ de sobrepeso e $14,3 \%$ de obesidade, entre os anos de 2008 e 2009. Entre os adolescentes (10 a 19 anos), a prevalência de obesidade foi de 4,9\% e a de sobrepeso de 20,5\% no mesmo período (IBGE, 2010a). 
Os impactos do excesso de peso englobam aumento do risco para o desenvolvimento de morbidades crônicas e surgimento precoce de doença cardiovascular. No que tange aos aspectos psíquicos e cognitivos, a obesidade pode predispor à ansiedade, depressão e distúrbios de sono. Como intervenções importantes para o enfrentamento da obesidade podemos citar a prática de atividades físicas e a redução do tempo de tela (SOCIEDADE BRASILEIRA DE PEDIATRIA, 2017).

A prevenção da obesidade pressupõe esforços integrados entre toda a rede de apoio da criança, qual seja, a família, a escola e a comunidade, além dos profissionais de saúde responsáveis por seu acompanhamento e demais setores sociais (SOCIEDADE BRASILEIRA DE PEDIATRIA, 2019).

Recentemente, o mundo se deparou com um grande desafio que modificou profundamente os hábitos de vida de diversas populações: a pandemia de COVID-19. As implicações desta súbita quebram de paradigmas são amplas e repercutem nos diversos âmbitos de interação social. Entre elas, a epidemia de obesidade precisa ser considerada e as implicações nesse cenário devem ser avaliadas. Um recorte possível seria a obesidade entre crianças e adolescentes, de várias formas favorecida durante o distanciamento social imposto como estratégia de enfretamento à disseminação da COVID-19 (SOCIEDADE BRASILEIRA DE PEDIATRIA, 2020).

Com a mobilidade restrita ao lar, a interrupção de aulas e de atividades grupais, a maior exposição às telas e modificações na alimentação, é necessário entender de que forma as medidas antropométricas dos mais jovens são alteradas e os possíveis impactos na epidemia de obesidade em decorrência da pandemia que ora se apresenta.

\section{REVISÃO BIBLIOGRÁFICA}

\section{Fatores que influenciam na gênese da obesidade infantil}

A obesidade é um problema de saúde pública tanto em países desenvolvidos quanto em países em desenvolvimento. Ela é uma doença multifatorial e, portanto, sofre influência de fatores biológicos, fisiológicos, metabólicos, psicológicos, comportamentais e socioeconômicos (OLIVEIRA AMA, et al., 2003).

A obesidade infantil, por sua vez, é um processo dinâmico, no qual aspectos comportamentais, cognitivos e emocionais convergem e agem mutuamente, tendo influência, assim, das atitudes parentais, do sedentarismo, do padrão nutricional e do estresse familiar (PUDER JJ e MUNSCH S, 2010).

Ao longo das últimas três décadas, o Brasil está vivendo uma transição demográfica, socioeconômica e, consequentemente, nutricional. Nos anos 50 , o país se caracterizava como fundamentalmente rural e a alimentação das pessoas se baseava na agricultura de subsistência (BATISTA FILHO M e RISSI A, 2003). O acesso ao alimento e à saúde eram escassos e, portanto, a desnutrição infantil era uma realidade. Com o êxodo rural, ocorreu a urbanização e, atualmente, o país se tornou majoritariamente urbano (IBGE, 2010b).

A partir dos anos 80 , com o processo de urbanização e a melhoria ao acesso à saúde, a taxa de desnutrição diminuiu. Ao contraponto, alimentos industrializados com alto teor de açúcar e sal, começaram a se tornar uma realidade no cotidiano do brasileiro. Dessa forma, o comportamento alimentar se alterou, caracterizando a transição alimentar. Essa transição teve início na década de $80 \mathrm{com}$ a redução da prevalência da desnutrição e aumento concomitante da obesidade (MAYER APF, 2011).

O novo hábito alimentar adotado por muitas pessoas em cidades grandes se caracteriza por uma alimentação rápida e prática devido ao ritmo acelerado do cotidiano recorrendo, em muitos casos, à alimentos pré-prontos e industrializados com baixa qualidade nutricional. O comportamento alimentar vivido pelos adultos, em muitos casos, é repassado para as crianças e adolescentes, e por isso o novo hábito observado tem caráter intergeracional. Dessa forma, crianças e adolescentes têm acesso à uma alimentação inadequada, exacerbando problemas como o sobrepeso e a obesidade (MAYER APF, 2011).

Ao longo do processo de urbanização, pôde-se observar também uma redução dos espaços recreativos e de áreas ao ar livre onde as pessoas e, principalmente, as crianças possam brincar e praticar esportes. Com isso, os infantes começaram a se exercitar menos e passar cada vez mais tempo expostos à aparatos 
eletrônicos, o que constitui importante estímulo ao sedentarismo. A maior prevalência de obesidade infantil e juvenil no Brasil, em concordância com os demais países, está condicionada a fatores socioeconômicos, condições familiares e estilo de vida atual (MAYER APF, 2011).

Um importante fator agravante à má alimentação infantil é o marketing da indústria alimentícia. Essa é uma questão internacionalmente discutida e alguns países estipulam que as propagandas devam conter alertas sobre os prejuízos do consumo exacerbado e devem ser restritas em horários de programação infantil (REIS CEG, VASCONCELOS IAL, BARROS JFN, 2011).

\section{Situação da obesidade em crianças e adolescentes no Brasil}

O excesso de peso é uma realidade global e sua prevalência aumenta progressivamente, ano após ano. Segundo a UNICEF (2019), entre os anos de 2000 e 2016, a proporção de crianças e adolescente com excesso de peso passou de 1 a cada 10 para 1 a cada 5 . O excesso de peso aumentou globalmente, enquanto a desnutrição, mesmo com acentuada redução, persiste como triste realidade nas áreas mais vulneráveis do mundo.

A Pesquisa de Orçamento Familiar (POF) de 2008-2009, revelou que 36,6\% das crianças no país têm excesso de peso. Em 1974, o Brasil tinha um percentual de 1,4\% de crianças obesas e em 2009 esse índice foi para $16,6 \%$. Entre os adolescentes, essa taxa era de $0,4 \%$ em 1974 e foi para 5,9\% em 2008 . Os indivíduos nessas condições são residentes, em sua maioria, no ambiente urbano e principalmente no sudeste brasileiro. (IBGE, 2008). Em 2015, o IBGE conduziu a 3ª edição da Pesquisa Nacional de Saúde do Escolar (PeNSE) e os resultados demonstram um percentual de $23,7 \%$ de adolescentes com excesso de peso. Destes, $7,8 \%$ eram obesos (IBGE, 2016).

Apesar da maior contribuição percentual na obesidade ser proveniente das metrópoles, áreas socioeconomicamente desfavorecidas também apresentam reflexos dessa mudança na dinâmica alimentar (PIMENTA, et al., 2015).

A má nutrição é uma realidade cada vez mais significativa em todo o mundo e envolve três aspectos: a subnutrição, a fome oculta e o sobrepeso. Em relação à obesidade e sobrepeso, a prevalência é ainda maior nos estratos mais desfavorecidos socioeconomicamente. Esse quadro é explicado pela maior insegurança alimentar e menor acesso aos alimentos de boa qualidade nutricional que são, geralmente, mais caros. Segundo a UNICEF (2019), 44\% das crianças entre 6 a 23 meses não ingere frutas ou legumes e 59\% não são alimentadas com ovos, laticínios, peixe ou carne.

O Ministério da Saúde (MS) conta com um plano de ações e estratégias de enfrentamento às doenças crônicas não transmissíveis que propõem metas para redução dessas doenças nos próximos 10 anos. Como o excesso de peso é importante fator de risco, sua redução é uma medida de prevenção e tornou-se uma meta desse plano. Assim, o MS pretende reduzir a obesidade entre as crianças de 5 a 9 anos, chegando à prevalência de $8 \%$ em 2022. Entre os adolescentes (10 a 19 anos), a meta é alcançar a prevalência de $3 \%$ em 2022. Entretanto, caso as medidas de prevenção e controle não sejam eficazes, a projeção é de que, em 2022 , cerca de $46,5 \%$ das crianças de 5 a 9 anos sejam obesas e, entre os adolescentes, este percentual será de 13,8\% (BRASIL, 2011).

\section{Impactos da obesidade na saúde das crianças e adolescentes}

O caráter multifatorial da obesidade implica na ocorrência de múltiplas repercussões. As alterações mais frequentemente citadas são as que dizem respeito à elevação do risco para o desenvolvimento de doenças crônicas e surgimento de prejuízos cardiovasculares precoces. No entanto, os impactos da obesidade na saúde infantil e juvenil vão além dos mencionados acometimentos físicos (CUNHA LM, et al., 2018).

Entre as implicações físicas, podemos destacar os distúrbios endócrinos, representados por resistência insulínica, diabetes mellitus tipo 2, hiperandrogenismo em meninas e anormalidades no crescimento Além destes, ocorrem prejuízos cardiovasculares, acompanhados de elevação da pressão arterial, dislipidemias, modificações estruturais e funcionais do coração e desenvolvimento e evolução precoces de placa aterosclerótica. A obesidade também está relacionada com problemas ortopédicos, como deformidades, 
fraturas, mialgias e, consequentemente, maiores prejuízos na mobilidade. Outras desordens acometem os sistemas hepático, renal, respiratório, tegumentar e nervoso (SOCIEDADE BRASILEIRA DE PEDIATRIA, 2019).

O Estudo de Riscos Cardiovasculares em Adolescentes (ERICA), avaliou 73.399 estudantes de 12 a 17 anos e encontrou prevalência de pré-hipertensão de $14,5 \%$ e prevalência de hipertensão arterial de $9,6 \%$, enquanto a prevalência de sobrepeso encontrada foi de $17,1 \%$ e a prevalência de obesidade foi de $8,4 \%$. A correlação de dados permitiu concluir que $17,8 \%$ da prevalência de hipertensão arterial pode ser atribuída à obesidade. Nas regiões Norte e Nordeste essa correlação mostrou-se mais presente, sendo identificada uma prevalência até seis vezes maior de hipertensão arterial em adolescentes obesos quando comparados com os não obesos (BLOCH KV, et al., 2016).

Além dos prejuízos físicos, é necessário considerar os possíveis prejuízos psicológicos decorrentes do excesso de peso, como desenvolvimento de depressão, ansiedade, distúrbios do sono, baixa autoestima, imagem corporal distorcida, rendimento escolar aquém do esperado e dificuldades de relacionamento (SOCIEDADE BRASILEIRA DE PEDIATRIA, 2019).

Um estudo realizado com crianças de um programa de acompanhamento multidisciplinar mostrou impactos negativos na imagem corporal e sentimento de culpa, tristeza e inadequação social provocados pelo excesso de peso (VICTORINO SVZ, et al., 2014). Outro estudo comparou a qualidade de vida entre crianças obesas e crianças com o peso adequado, encontrando escores superiores nas últimas, em todas as comparações (CUNHA LM, et al., 2018).

Uma pesquisa encontrou associação entre obesidade e estresse em quase metade das crianças avaliadas, sendo maior a frequência entre meninas (BERTOLETTI J e GARCIA-SANTOS SC, 2012). Todos esses estudos tiveram seus resultados limitados pelo tamanho da amostra, reforçando a necessidade de estudos de base populacional para estimar a ocorrência dos fatores associados à obesidade, principalmente em crianças.

Em relação aos adolescentes, dados do PeNSE de 2015 mostram que 84,1\% dos estudantes brasileiros do $9^{\circ}$ ano consideram que a própria imagem corporal é importante ou muito importante. Cerca de $11,6 \%$ dos adolescentes do sexo masculino declararam estar insatisfeitos com o próprio corpo, enquanto nas meninas, esse percentual atinge $23,3 \%$. A prevalência de estudantes que se achavam "gordos" ou "muito gordos" era de $18,3 \%$ (IBGE, 2016).

As intensas mudanças corporais da puberdade impõem ao adolescente adaptações constantes. Nessa faixa etária, o corpo adquire um significado simbólico importante que, muitas vezes, é capaz de determinar o pertencimento, a aceitação e o relacionamento entre pares. (BRASIL, 2006). Ainda de acordo com o PeNSE de $2015,7 \%$ dos estudantes do $9^{\circ}$ ano declararam ter induzido o vômito ou utilizado laxante com o intuito de perder peso nos 30 dias que antecederam a coleta de dados da pesquisa, sendo prática mais frequente entre as meninas $(7,5 \%)$ do que entre os meninos $(6,5 \%)$.

Em relação ao uso de medicamento, fórmula ou outro produto com o objetivo de ganhar massa muscular, foi encontrada uma prevalência de 8,6\% entre os meninos e de 5,6\% entre as meninas (IBGE, 2016). Portanto, no acompanhamento de adolescentes com excesso de peso é necessário identificar comportamentos que podem comprometer seriamente a saúde e propor intervenções assertivas no sentido de minimizá-las.

\section{Prevenção e controle da obesidade infantil}

Em decorrência das diversas implicações atribuídas ao distúrbio da obesidade, faz-se necessário abordar, além das consequências, as estratégias de prevenção e controle dessa morbidade. Uma estratégia significativa para o enfrentamento da obesidade infantil é a associação entre atividade física e melhoria da qualidade alimentícia, hábitos eficazes para alcançar o equilíbrio metabólico (BRAVIN MB, et al., 2015). A atividade física reduz a gordura visceral, atenua fatores de risco, fortalece ossos e músculos, melhora condicionamento cardiorrespiratório, reduz estresse, aumenta autoestima, previne depressão, melhora o sono e controla peso, pressão sanguínea e níveis de glicose (MINISTÉRIO DA SAÚDE, 2017). 
A obesidade infantil é um problema familiar, não individual. Os hábitos familiares contribuem para o estado nutricional das crianças e, até certo ponto, dos adolescentes, por isso, diante de um quadro de sobrepeso ou obesidade, a família precisa participar das estratégias para alcançar o peso adequado (NOGUEIRA E, et al., 2020). A escola também é crucial para a prevenção e controle da obesidade pois, geralmente, introduz e incentiva a prática de atividades físicas, além de ser uma promotora de educação em saúde (TESTA WL, POETA LS, DA SILVA DUARTE MF, 2017).

A escola também é um espaço importante quando consideramos a nutrição, pois as que oferecem merenda geralmente seguem padrões nutricionais adequados. Entretanto, escolas que possuem ponto de venda alternativo de alimentos podem oferecer possibilidades pouco nutritivas, contribuindo para a elevação dos índices de sobrepeso e obesidade (MAYER APF, 2011). Crianças com melhores condições socioeconômicas, possuem maior acesso aos alimentos hipercalóricos que, associados ao maior tempo de tela, contribuem para o avanço da obesidade entre estes indivíduos. (TEIXEIRA AS, DESTRO DS, 2010). O valor elevado dos alimentos mais nutritivos, por sua vez, torna o excesso de peso um importante problema também nas classes menos favorecidas.

Na primeira infância, a alimentação da criança está praticamente restrita às orientações dos pais e da escola, sendo período privilegiado para o estabelecimento de estratégias que promovam hábitos alimentares saudáveis com repercussões, inclusive, a longo prazo (PAES ST, MARINS JCB, ANDREAZZI AE, 2015).

A obesidade gera prejuízos que podem se refletir na vida adulta. Sendo assim, a prevenção e controle da obesidade ainda na infância, tem papel imprescindível para melhoria da qualidade de vida e atenuação dos problemas físicos e psíquicos que impactam em vários âmbitos da vida desses indivíduos (SULZBACH EAG, DAL BOSCO SM, 2012).

\section{Possíveis impactos do distanciamento social imposto pela pandemia do COVID-19 na epidemia de obesidade infantil}

O COVID-19 vem assolando o mundo, ultrapassando fronteiras e atingindo pessoas de diversas classes e idades. O contato, o toque e a convivência estão restritos devido à alta transmissibilidade viral (RACHE B, et al. 2020). Na atual perspectiva, não há previsão de retorno à antiga rotina. Diversas atividades que implicam aglomeração de pessoas, como as aulas presenciais, estão paralisadas, exigindo de todos os envolvidos a criação de estratégias para reinventar a educação e permitir que ela alcance seu papel da forma mais inclusiva possível (ORNELL F, et al., 2020).

A interrupção das aulas presenciais, além de todos os possíveis impactos na formação dos estudantes, gera também uma lacuna no que se refere à alimentação, já que a merenda escolar é fonte segura e equilibrada de alimentação, sendo, em alguns casos, a única refeição com essas características acessível a vários alunos. Na situação econômica atual, o sustento de muitas famílias ficou comprometido e a disponibilidade de alimentos nutritivos ficou ainda mais escassa para as crianças e adolescentes em situação de vulnerabilidade socioeconômica. Nessas famílias, a contribuição da escola na alimentação fica ainda mais evidenciada e a impossibilidade de desfrutá-la pode gerar prejuízos no crescimento e desenvolvimento de inúmeras crianças e adolescentes (ORNELL F, et al., 2020).

As estratégias para conter a disseminação da COVID-19 impactam diretamente na alimentação, já que a restrição da mobilidade limita a frequência de compras de gêneros alimentícios. A consequência é um maior consumo de alimentos processados e enlatados que são mais fáceis de adquirir e armazenar e possuem maior prazo de validade. Esse tipo de alimento tem baixo valor nutricional e alto valor calórico, contribuindo para o surgimento ou agravamento de sobrepeso e obesidade em crianças e adolescentes (SOCIEDADE BRASILEIRA DE PEDIATRIA, 2020).

O distanciamento social também gerou repercussões nas relações familiares. As crianças e adolescentes que passavam grande parte do dia nas creches e escolas, estão restritas ao lar em período integral. Nesse cenário, o estresse e a sobrecarga do cuidador podem ocasionar uma exacerbação da agressividade e violência (MARQUES ES, et al., 2020). O aumento da exposição à violência psicológica, o cenário de incertezas, o afastamento da rotina e de pessoas próximas geram importantes reflexos na saúde mental das crianças e dos adolescentes (MINITERIO DE SALUD Y PROTECCÍON SOCIAL, 2020). 
Considerando que a obesidade tem forte associação com a ocorrência de ansiedade e depressão, é necessário acompanhar de perto possíveis alterações comportamentais de crianças e adolescentes obesos, principalmente pela associação de fatores inerentes ao distanciamento social que podem provocar alterações de humor (SOCIEDADE BRASILEIRA DE PEDIATRIA, 2020).

Outro fator que impacta diretamente na epidemia de obesidade é a redução das atividades físicas, fortemente influenciada pela interrupção das aulas presenciais, já que a escola é ambiente propício para as interações grupais ativas, realização de esportes e atividades recreativas. Além disso, muitas crianças não dispõem de local adequado para brincadeiras ou realizações de atividades que permitam a cessação do sedentarismo (CABRERA MPC, AGUILAR JLL, JARAMILLO LMT, 2020). As aulas de apoio e as academias estão com atividades suspensas o que também contribui para a manutenção de altos níveis de inatividade (SOCIEDADE BRASILEIRA DE PEDIATRIA, 2020).

A obesidade está comprovadamente relacionada à desregulação da resposta imune, tornando o indivíduo susceptível a infecções. Em pacientes com COVID-19, a obesidade pode causar problemas respiratórios e dificuldade de intubação traqueal, aumentando risco de complicações e mortalidade (SOCIEDADE BRASILEIRA DE PEDIATRIA, 2020).

Deste modo, para evitar o desenvolvimento ou agravamento da obesidade e evitar as complicações decorrentes dela, a família deve proporcionar um ambiente saudável e seguro, fornecendo apoio emocional, priorizando alimentos nutritivos, estimulando as atividades físicas e limitando o tempo de tela. Essas medidas são necessárias tanto para evitar o excesso de peso e suas repercussões à longo prazo, quanto para suplantar o aumento do risco associado à obesidade caso essas crianças e adolescentes sejam expostos à infecção pelo COVID-19 (SOCIEDADE BRASILEIRA DE PEDIATRIA, 2020).

\section{CONSIDERAÇÕES FINAIS}

O caráter epidêmico da obesidade em crianças e adolescentes revela-se um complexo desafio que exige abordagem multidisciplinar e integral. $O$ aumento da prevalência do excesso de peso e sua atuação como fator de risco para a ocorrência de diversas morbidades projeta repercussões importantes na dinâmica dos serviços de saúde, no bem-estar social e produtividade durante a idade adulta. Encarar o enfrentamento do sobrepeso e da obesidade como prioridade em saúde e conceber que a prevenção em tenra idade é mais assertiva que as intervenções na vida adulta, subsidiam a busca pelo bem-estar e desenvolvimento saudável das crianças e adolescentes mesmo diante da insegurança e da incerteza que imperam no atual cenário mundial.

\section{REFERÊNCIAS}

1. ALVES JM, et al. Ensinar e aprender em tempo de COVID-19: entre o caos e a redenção, 2020.

2. BATISTA FILHO M, RISSIN A. A transição nutricional no Brasil: tendências regionais e temporais. Cadernos de Saúde pública, Rio de Janeiro, 2003; 19(1): 181-191.

3. BERTOLETTI J, GARCIA-SANTOS SC. Avaliação do Estresse na Obesidade Infantil. Psico, 2012; 43(1): 32-38.

4. BLOCH KV, et al. ERICA: prevalência de hipertensão arterial e obesidade em adolescentes brasileiros. Revista de Saúde Pública. Rio de Janeiro, 2016; 50(1): 9s.

5. BRASIL. Cadernos de Atenção Básica, n. 12. Ministério da Saúde. Secretaria de Atenção à Saúde. Departamento de Atenção Básica. Brasília: Ministério da Saúde, 2006; 108p.

6. BRASIL. Plano de ações estratégicas para o enfrentamento das doenças crônicas não transmissíveis (DCNT) no Brasil 2011-2022. Ministério da Saúde. Secretaria de Vigilância em Saúde. Departamento de Análise de Situação de Saúde. Brasília: Ministério da Saúde, 2011.160 p.

7. BRASIL. Política Nacional de Alimentação e Nutrição. Ministério da Saúde. Secretaria de Atenção à Saúde. Departamento de Atenção Básica. Brasília: Ministério da Saúde, 2013; 84p.

8. BRASIL. Vigitel Brasil 2018: vigilância de fatores de risco e proteção para doenças crônicas por inquérito telefônico: estimativas sobre frequência e distribuição sociodemográfica de fatores de risco e proteção para doenças crônicas nas capitais dos 26 estados brasileiros e no Distrito Federal em 2018. Ministério da Saúde, Secretaria de Vigilância em Saúde, Departamento de Análise em Saúde e Vigilância de Doenças não Transmissíveis. Brasília: Ministério da Saúde, 2019; 132p.

9. BRAVIN MB, et al. A influência do exercício físico na obesidade infantil. Revista Ciência e Estudos Acadêmicos de Medicina. Cáceres, 2015; 1(4):37-51. 
10. CABRERA MPC, et al. La colisión de dos pandemias: covid-19 y obesidad. 2020.

11. CUNHA LM, et al. Impacto negativo da obesidade sobre a qualidade de vida de crianças. Revista Brasileira de Obesidade, Nutrição e Emagrecimento, 2018; 70(12): 231-238.

12. INSTITUTO BRASILEIRO DE GEOGRAFIA E ESTATÍSTICA. Pesquisa de Orçamentos Familiares (POF) 2008-2009. Antropometria e estado nutricional de crianças, adolescentes e adultos no Brasil. Rio de Janeiro: IBGE, $2010 \mathrm{a}$.

13. INSTITUTO BRASILEIRO DE GEOGRAFIA E ESTATÍSTICA. População residente por situação de domicilio. Rio de Janeiro: IBGE, 2010b.

14. INSTITUTO BRASILEIRO DE GEOGRAFIA E ESTATÍSTICA. Pesquisa nacional de saúde do escolar: 2015. Coordenação de População e Indicadores Sociais. Rio de Janeiro, 2016. 132 p.

15. MARQUES ES, et al. A violência contra mulheres, crianças e adolescentes em tempos de pandemia pela COVID-19: panorama, motivações e formas de enfrentamento. Cadernos de Saúde Pública, 2020; 36.

16. MAYER APF. As relações entre família, escola e desenvolvimento do sobrepeso e obesidade infantil. Dissertação (Pós-graduação em Educação). Faculdade Federal do Paraná, Curitiba, 2011.

17. MINISTÉRIO DA SAÚDE. Benefícios da atividade física. 2017. Disponível em: < saude.gov.br/artigos/781-atividadesfisicas/40394-beneficios-da-atividade-fisica >. Acesso em: 20 jun. 2020.

18. MINITERIO DE SALUD Y PROTECCÍON SOCIAL. Orientaciones para la salida de niñas, niños y adolescentes a espacio público durante la pandemia por covid-19 en colombia, 2020.

19. NOGUEIRA E, et al. A obesidade infantil no Brasil e fatores associados: desafios para os professores de educação física. Revista Internacional de apoyo a la inclusión, logopedia, sociedad y multiculturalidad, 2020 6(1): 13-24.

20. OLIVEIRA AMA, et al. Sobrepeso e obesidade infantil: influência de fatores biológicos e ambientais em Feira de Santana, BA. Arquivo Brasileiros de Endocrinologia e Metabologia. São Paulo, 2003; 47(2): 144-150.

21. ORGANIZAÇÃO DAS NAÇÕES UNIDAS. Transformando nosso mundo: A agenda 2030 para o desenvolvimento sustentável. Centro de Informações das Nações Unidas para o Brasil (UNIC Rio), 2015.

22. ORNELL F, et al. Pandemia de medo e COVID-19: impacto na saúde mental e possíveis estratégicas. Revista debates in psychiatry. Porto Alegre, 2020.

23. PAES ST, et al. Efeitos metabólicos do exercício físico na obesidade infantil: uma visão atual. Revista Paulista de Pediatria, 2015,33(1): 122-129.

24. PIMENTA TAM, et al. Políticas públicas de intervenção na obesidade infantil no Brasil: uma breve análise da política nacional de alimentação e nutrição e política nacional de promoção da saúde. Journal of Health Sciences, 2015, 17(2).

25. PUDER, JJ, MUNSCH S. Psychological correlates of childhood obesity. International Journal of Obesity, 2010; 34: 3743.

26. RACHE B, et al. Necessidades de infraestrutura do SUS em preparo à COVID-19: leitos de UTI, respiradores e ocupação hospitalar. São Paulo: Instituto de Estudos para Políticas de Saúde, 2020, (3): 1-5

27. REIS CEG, et al. Políticas públicas de nutrição para o controle da obesidade infantil. Revista paulista de pediatria, 2011, 29(4): 625-633.

28. SOCIEDADE BRASILEIRA DE PEDIATRIA. Obesidade em Crianças e Adolescentes e COVID-19. Nota de Alerta. Sociedade Brasileira de Pediatria. Grupo de Trabalho em Atividade Física. São Paulo: SBP, 2020.

29. SOCIEDADE BRASILEIRA DE PEDIATRIA. Obesidade na infância e adolescência: Manual de Orientação. Sociedade

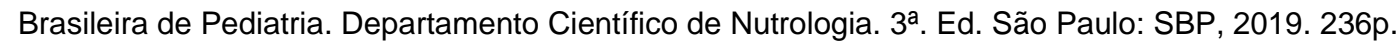

30. SOCIEDADE BRASILEIRA DE PEDIATRIA. Promoção da Atividade Física na Infância e Adolescência: Manual de Orientação. Sociedade Brasileira de Pediatria. Grupo de Trabalho em Atividade Física. São Paulo: SBP, 2017. 14p.

31. SULZBACH EAG, DAL BOSCO SM. Obesidade infantil-uma revisão bibliográfica. Revista Destaques Acadêmicos, 2012, 4(3): 113-127.

32. TEIXEIRA AS, DESTRO DS. Obesidade infantil e educação física escolar: Possibilidades pedagógicas. Revista Eletrônica da Faculdade Metodista Granbery, 2010,9:1-15.

33. TESTA WL, et al. Exercício físico com atividades recreativas: uma alternativa para o tratamento da obesidade infantil. RBONE-Revista Brasileira De Obesidade, Nutrição E Emagrecimento, 2017,11(62):49-55.

34. UNICEF. Situação Mundial da Infância 2019: Crianças, alimentação e nutrição. Sumário Executivo. UNICEF Office of Global Insight and Policy 3 United Nations Plaza: New York, 2019. 24p.

35. VICTORINO SVZ, et al. Viver com obesidade infantil: a experiência de crianças inscritas em programa de acompanhamento multidisciplinar. Revista Rene, Fortaleza, 2014; 15(6): 980-989. 